\title{
Uniform Stability of a Class of Fractional-Order Nonautonomous Systems with Multiple Time Delays
}

\author{
Tao Zou, Jianfeng Qu, Yi Chai, Maoyun Guo, and Congcong Liu
}

School of Automation, Chongqing University, Chongqing 400044, China

Correspondence should be addressed to Jianfeng Qu; qujianfeng@cqu.edu.cn

Received 8 July 2013; Revised 23 December 2013; Accepted 25 December 2013; Published 12 February 2014

Academic Editor: Reinaldo Martinez Palhares

Copyright (C) 2014 Tao Zou et al. This is an open access article distributed under the Creative Commons Attribution License, which permits unrestricted use, distribution, and reproduction in any medium, provided the original work is properly cited.

In mathematics, to a large extent, control theory addresses the stability of solutions of differential equations, which can describe the behavior of dynamic systems. In this paper, a class of fractional-order nonautonomous systems with multiple time delays modeled by differential equations is considered. A sufficient condition is established for the existence and uniqueness of solutions for such systems involving Caputo fractional derivative, and the uniform stability of solution is studied. At last, two examples are given to demonstrate the applicability of our results.

\section{Introduction}

Fractional calculus is an ongoing topic for more than 300 years; it is a generalization of ordinary differentiation and integration to arbitrary (noninteger) order. The number of literature concerning the application of fractional calculus has been growing rapidly, especially in recent years. In fact, fractional derivatives are more adequate to describe underlying phenomena than traditionally used integral-order derivatives in many cases, because fractional-order derivatives provide an excellent tool for the description of memory and hereditary properties of various processes, in contrast to integral-order derivatives. Nowadays, fractional calculus is a flourishing field of active research [1-9].

Stability is one of the most fundamental and interesting problem in control theory. So far, there have been some advances in control theory of fractional dynamical systems for stability questions such as robust stability [10-12], MittagLeffler stability [13], bounded-input bounded-output stability $[14,15]$, uniform stability [16], finite-time stability [17], and robust controllability [18]. However, it should be noted that it is difficult to evaluate the stability for fractionalorder dynamic systems by simply examining its characteristic equation either by finding its dominant roots or by using other algebraic methods. In addition, it is well known that Lyapunov direct method cannot be simply extended and applied to the case of fractional order, although many stability results about integer-order systems are obtained by constructing a suitable Lyapunov functional [19-22]. From the above discussion, it may be seen that study on the stability of fractional-order systems is still meaningful. Moreover, time delay plays an important role in mathematical modeling of many real world phenomena. Time delay can have an effect on the stability of a system and occasionally can cause a system to become unstable. To the best of our knowledge, there are relatively few results on the stability of fractionalorder systems with delay, such as Lazarević and Spasić [17], Akbari Moornani and Haeri [23], Kumar and Sukavanam [24], Wang et al. [25], and El-Sayed and Gaafar [26]. In [17], a finite-time stability test procedure is proposed for linear nonhomogeneous fractional-order systems with a pure time delay. In [23], two theorems are given to check the robust BIBO stability of two large classes of fractional-order delay systems (retarded and neutral types), respectively. In [24], sufficient conditions are established for the approximate controllability of a class of semilinear delay control systems of fractional order. A delayed fractional-order financial system is proposed and the complex dynamical behaviors of such a system are discussed by numerical simulations in [25]. The existence of a unique solution and the uniform stability of solution are proved for a class of nonlinear nonautonomous system of Riemann-Liouville fractional differential systems 
with different constant delays and nonlocal condition in [26]. For more details, the reader can refer to [27-30].

Motivated by the above discussions, this paper aims at studying the uniform stability of a class of fractional-order nonautonomous systems with multiple time delays. Besides, a sufficient condition for the existence and uniqueness of solutions is given.

The rest of this paper is organized as follows. In Section 2, some useful definitions, lemmas, and notations are introduced. In Section 3, the existence and uniqueness of solutions and uniform stability problem for fractional-order nonautonomous systems with multiple time delays are studied. In Section 4, two numerical examples are presented to demonstrate the main results. Finally, some conclusions are drawn in Section 5.

\section{Preliminaries}

In this section, we present some definitions, lemmas, and notations related to the main results that we will obtain in the following.

Definition 1 (see [31]). The fractional-order integral of a function $f(t)$ of order $\alpha \in R^{+}$is defined by

$$
I_{t_{0}}^{\alpha} f(t)=\frac{1}{\Gamma(\alpha)} \int_{t_{0}}^{t} \frac{f(\tau)}{(t-\tau)^{1-\alpha}} d \tau
$$

where $\Gamma(\cdot)$ is the gamma function given as

$$
\Gamma(z)=\int_{0}^{\infty} t^{z-1} e^{-t} d t .
$$

Definition 2 (see [32]). The Caputo fractional derivative $D^{\alpha}$ of order $\alpha$ of a function $f(t)$ is defined as

$$
D_{t_{0}}^{\alpha} f(t)=\frac{1}{\Gamma(n-\alpha)} \int_{t_{0}}^{t} \frac{f^{(n)}(\tau)}{(t-\tau)^{\alpha+1-n}} d \tau, \quad(n-1<\alpha<n),
$$

where $n=[\alpha],[\alpha]$ is the ceiling function.

From the previous definitions, it is recognized that fractional derivative represents a global property of a function within a given closed interval $[0, t]$, while integral derivative of a function is only related to its nearby value of the independent variable, which is a local property.

Lemma 3 (see [32]). Let $n$ be a positive integer such that $n-1<$ $\alpha<n$; if $f(t) \in C^{n-1}\left[t_{0}, t\right]$, then

$$
I_{t_{0}}^{\alpha} D_{t_{0}}^{\alpha} f(t)=f(t)-\sum_{m=0}^{n-1} \frac{f^{(m)}\left(t_{0}\right)}{m !}\left(t-t_{0}\right)^{m} .
$$

In particular, if $0<\alpha<1$ and $f(t) \in C\left[t_{0}, t\right]$, then

$$
I_{t_{0}}^{\alpha} D_{t_{0}}^{\alpha} f(t)=f(t)-f\left(t_{0}\right)
$$

The following notations will be used throughout the paper. Let $C\left([a, b], R^{n}\right)$ denote the class of all continuous column $n$-vectors function defined on $[a, b]$; for $x \in$ $C\left([a, b], R^{n}\right)$, the norm is $\|x(t)\|=\sum_{i=1}^{n} \sup _{t \in(a, b]}\left\{e^{-K t}\left|x_{i}(t)\right|\right\}$, where $K$ is a large enough constant. In addition, we define the norm of $A=\left(a_{i j}\right)_{n \times n}$ by $\|A\|=\sum_{i=1}^{n} a_{i}=\sum_{i=1}^{n} \sup _{t, \forall j}\left|a_{i j}(t)\right|$.

\section{Main Results}

The differential equation describing the dynamic behavior of a class of nonautonomous systems with multiple time delays can be represented as follows:

$$
\begin{aligned}
D^{\alpha} x_{i}(t)= & \sum_{j=1}^{n} a_{i j}(t) x_{j}(t)+\sum_{j=1}^{n} b_{i j}(t) f_{j}\left(x_{j}(t)\right) \\
& +\sum_{j=1}^{n} c_{i j}(t) f_{j}\left(x_{j}\left(t-\tau_{i j}\right)\right) \\
& +u_{i}(t), \quad t \in[0, T], \quad i \in N=\{1,2, \ldots n\},
\end{aligned}
$$

where $T<+\infty ; D^{\alpha}$ denotes Caputo fractional-order derivative of order $\alpha(0<\alpha<1)$ with $t_{0}=0$; $x(t)=$ $\left(x_{1}(t), x_{2}(t), \ldots, x_{n}(t)\right)^{T}$ represents the state vector at time $t$; $A(t)=\left(a_{i j}\right)_{n \times n}, B(t)=\left(b_{i j}\right)_{n \times n}, C(t)=\left(c_{i j}\right)_{n \times n}$, and $u(t)=$ $\left(u_{i}(t)\right)_{n \times 1}$ are given matrices whose elements are absolutely continuous; $f(x(t))=\left(f_{1}\left(x_{1}(t)\right), f_{2}\left(x_{2}(t)\right), \ldots, f_{n}\left(x_{n}(t)\right)\right)$ corresponds to a vector function; $\tau_{i j}$ is a constant and denotes the corresponding time delay.

Accompanying the system (6) is an initial condition of the form:

$$
x_{i}(t)=\phi_{i}(t), \quad t \in[-\tau, 0]
$$

for all $i \in N$, where $\tau=\max _{i, j \in N}\left\{\tau_{i j}\right\}$ and $\phi_{i}(t)$ is continuous on $[-\tau, 0]$.

In order to prove the main results, we make the following assumption:

(H1) $f_{j}(j=1,2, \ldots, n)$ is Lipschitz continuous with Lipschitz constant $L_{j}$; that is,

$$
\left|f_{j}(x)-f_{j}(y)\right| \leq L_{j}|x-y|
$$

3.1. Existence and Uniqueness of Solutions. In this section, we will give a sufficient condition for the existence and uniqueness of solutions of system (6).

Theorem 4. Assume that (H1) holds; then, system (6) exists a unique solution $x(t)$ which satisfies $x(t) \in C\left([0, T], R^{n}\right)$ on $[0, T]$ and coincides with $\phi$ for $t \in[-\tau, 0]$. 
Proof. Equation (6) is equivalent to the equation

$$
\begin{aligned}
x_{i}(t)=\phi_{i}(0) & +\frac{1}{\Gamma(\alpha)} \\
\times \int_{0}^{t}(t-s)^{\alpha-1} & \\
\times & {\left[\sum_{j=1}^{n} a_{i j}(s) x_{j}(s)+\sum_{j=1}^{n} b_{i j}(s) f_{j}\left(x_{j}(s)\right)\right.} \\
& +\sum_{j=1}^{n} c_{i j}(s) f_{j}\left(x_{j}\left(s-\tau_{i j}\right)\right) \\
& \left.+u_{i}(s)\right] d s, \quad t \in[0, T] .
\end{aligned}
$$

Now, construct a mapping $T_{i}$, defined by

$$
\begin{aligned}
T_{i} x_{i}(t)=\phi_{i}(0) & +\frac{1}{\Gamma(\alpha)} \\
\times \int_{0}^{t}(t-s)^{\alpha-1} & \\
\times & {\left[\sum_{j=1}^{n} a_{i j}(s) x_{j}(s)+\sum_{j=1}^{n}\left(b_{i j}(s)+c_{i j}(s)\right)\right.} \\
& \left.\times f_{j}\left(x_{j}(s)\right)+u_{i}(s)\right] d s, \quad t \in[0, T],
\end{aligned}
$$

where $T x=\left(T_{1} x_{1}, T_{2} x_{2}, \ldots, T_{n} x_{n}\right)^{T}$.

For any two different $x(t), y(t) \in C\left([0, T], R^{n}\right)$, we have

$$
\begin{aligned}
& \left|T_{i} x_{i}(t)-T_{i} y_{i}(t)\right| \\
& \leq \frac{1}{\Gamma(\alpha)} \int_{0}^{t}(t-s)^{\alpha-1} \\
& \times\left[\sum_{j=1}^{n}\left|a_{i j}(s)\right|\left|x_{j}(s)-y_{j}(s)\right|\right. \\
& \quad+\sum_{j=1}^{n}\left(\left|b_{i j}(s)\right|+\left|c_{i j}(s)\right|\right) \\
& \left.\quad \times\left|f_{j}\left(x_{j}(s)\right)-f_{j}\left(y_{j}(s)\right)\right|\right] d s \\
& \leq \frac{1}{\Gamma(\alpha)} \int_{0}^{t}(t-s)^{\alpha-1} \\
& \times\left[\sum_{j=1}^{n}\left|a_{i j}(s)\right|\left|x_{j}(s)-y_{j}(s)\right|\right.
\end{aligned}
$$

$$
\begin{aligned}
& +\sum_{j=1}^{n}\left(\left|b_{i j}(s)\right|+\left|c_{i j}(s)\right|\right) \\
& \left.\times L_{j}\left|x_{j}(s)-y_{j}(s)\right|\right] d s,
\end{aligned}
$$

from which it follows that

$$
\begin{aligned}
& e^{-K t}\left|T_{i} x_{i}(t)-T_{i} y_{i}(t)\right| \\
& \leq \frac{1}{\Gamma(\alpha)} e^{-K t} \\
& \quad \times \int_{0}^{t}(t-s)^{\alpha-1} \\
& \quad \times\left[\sum_{j=1}^{n}\left|a_{i j}(s)\right|\left|x_{j}(s)-y_{j}(s)\right|\right. \\
& \quad+\sum_{j=1}^{n}\left(\left|b_{i j}(s)\right|+\left|c_{i j}(s)\right|\right) \\
& \left.\quad \times L_{j}\left|x_{j}(s)-y_{j}(s)\right|\right] d s
\end{aligned}
$$$$
\leq \frac{1}{\Gamma(\alpha)} \int_{0}^{t}(t-s)^{\alpha-1}
$$$$
\times\left[\sum_{j=1}^{n}\left|a_{i j}(s)\right| e^{-K(t-s)}\right.
$$$$
\times e^{-K s}\left|x_{j}(s)-y_{j}(s)\right|
$$$$
+\sum_{j=1}^{n}\left(\left|b_{i j}(s)\right|+\left|c_{i j}(s)\right|\right)
$$$$
\times L_{j} e^{-K(t-s)}
$$$$
\left.\times e^{-K s}\left|x_{j}(s)-y_{j}(s)\right|\right] d s
$$$$
\leq \frac{1}{\Gamma(\alpha)} \sup _{t, \forall j}\left|a_{i j}(t)\right|
$$$$
\times \int_{0}^{t}(t-s)^{\alpha-1}
$$$$
\times\left[\sum_{j=1}^{n} e^{-K(t-s)} e^{-K s}\left|x_{j}(s)-y_{j}(s)\right|\right] d s
$$ 


$$
\begin{aligned}
& +\frac{\left(\sup _{t, \forall j}\left|b_{i j}(t)\right|+\sup _{t, \forall j}\left|c_{i j}(t)\right|\right) L}{\Gamma(\alpha)} \\
& \times \int_{0}^{t}(t-s)^{\alpha-1} \\
& \quad \times\left[\sum_{j=1}^{n} e^{-K(t-s)} e^{-K s}\left|x_{j}(s)-y_{j}(s)\right|\right] d s \\
& \leq a_{i} \sum_{j=1}^{n} \sup _{t}\left\{e^{-K t}\left|x_{j}(t)-y_{j}(t)\right|\right\} \\
& \quad \times \frac{1}{\Gamma(\alpha)} \int_{0}^{t}(t-s)^{\alpha-1} e^{-K(t-s)} d s \\
& +\left(b_{i}+c_{i}\right) L \sum_{j=1}^{n} \sup _{t}\left\{e^{-K t}\left|x_{j}(t)-y_{j}(t)\right|\right\} \\
& \quad \times \frac{1}{\Gamma(\alpha)} \int_{0}^{t}(t-s)^{\alpha-1} e^{-K(t-s)} d s \\
& <\frac{a_{i}+\left(b_{i}+c_{i}\right) L}{K^{\alpha}}\|x(t)-y(t)\| .
\end{aligned}
$$

Then, we have

$$
\begin{aligned}
\| T x(t) & -T y(t) \| \\
= & \sum_{i=1}^{n} \sup _{t}\left\{e^{-K t}\left|T_{i} x_{i}(t)-T_{i} y_{i}(t)\right|\right\} \\
< & \sum_{i=1}^{n} \frac{a_{i}+\left(b_{i}+c_{i}\right) L}{K^{\alpha}}\|x(t)-y(t)\| \\
& =\frac{\|A\|+(\|B\|+\|C\|) L}{K^{\alpha}}\|x(t)-y(t)\| .
\end{aligned}
$$

For $K$ is a large enough constant, here we can choose $K$ such that $\|A\|+(\|B\|+\|C\|) L<K^{\alpha}$; then, we have

$$
\|T x(t)-T y(t)\|<\|x(t)-y(t)\| .
$$

From the above proof, we know that $T$ is a contraction mapping and it has a unique fixed point $x=T x$, so that system (6) exists a unique solution.

3.2. Stability of Nonautonomous Systems. In this section, we study the stability of solution of system (6).

Definition 5 (see [33]). The solution of (6) is called stable if for any $\varepsilon>0, t_{0} \geq 0$, there exists $\delta\left(\varepsilon, t_{0}\right)$ such that $t \geq t_{0} \geq 0$, $\|\varphi(t)-\phi(t)\|<\delta\left(\varepsilon, t_{0}\right)$ imply $\left\|y\left(t, t_{0}, \varphi\right)-x\left(t, t_{0}, \phi\right)\right\|<\varepsilon$ for any two solutions $x\left(t, t_{0}, \phi\right)$ and $y\left(t, t_{0}, \varphi\right)$. And the solution of (6) is called uniformly stable if $\delta\left(\varepsilon, t_{0}\right)$ can be chosen independently of $t_{0}: \delta\left(\varepsilon, t_{0}\right) \equiv \delta(\varepsilon)$.

Theorem 6. Under assumption (H1), the solution of system (6) is uniformly stable.
Proof. Let $x(t)=\left(x_{1}(t), x_{2}(t), \ldots, x_{n}(t)\right)^{T}$ be a solution of (6) with initial condition $x(t)=\phi(t) \in C\left([-\tau, 0], R^{n}\right)$, and we have

$$
\begin{aligned}
x_{i}(t)=\phi_{i}(0) & +\frac{1}{\Gamma(\alpha)} \\
\times \int_{0}^{t}(t-s)^{\alpha-1} & \\
\times & {\left[\sum_{j=1}^{n} a_{i j}(s) x_{j}(s)+\sum_{j=1}^{n} b_{i j}(s) f_{j}\left(x_{j}(s)\right)\right.} \\
& +\sum_{j=1}^{n} c_{i j}(s) f_{j}\left(x_{j}\left(s-\tau_{i j}\right)\right) \\
& \left.+u_{i}(s)\right] d s, \quad t \in[0, T] .
\end{aligned}
$$

And let $y(t)=\left(y_{1}(t), y_{2}(t), \ldots, y_{n}(t)\right)^{T}$ be a different solution with initial condition $y(t)=\varphi(t) \in C\left([-\tau, 0], R^{n}\right)$; then,

$$
\begin{aligned}
& \left|x_{i}(t)-y_{i}(t)\right| \\
& \leq\left|\phi_{i}(0)-\varphi_{i}(0)\right|+\frac{1}{\Gamma(\alpha)} \\
& \quad \times \int_{0}^{t}(t-s)^{\alpha-1} \\
& \times\left[\sum_{j=1}^{n}\left|a_{i j}(s)\right|\left|x_{j}(s)-y_{j}(s)\right|\right. \\
& \quad+\sum_{j=1}^{n}\left|b_{i j}(s)\right| \\
& \quad \times \sum_{j=1}^{n}\left|c_{i j}(s)\right| \mid f_{j}\left(x_{j}\left(s-\tau_{i j}\right)\right) \\
& \leq\left|\phi_{i}(0)-\varphi_{i}(0)\right|+\frac{1}{\Gamma(\alpha)} \\
& \times \int_{0}^{t}(t-s)^{\alpha-1} \\
& \times\left[\sum_{j=1}^{n}\left|a_{i j}(s)\right|\left|x_{j}(s)-y_{j}(s)\right|\right.
\end{aligned}
$$


Journal of Applied Mathematics

5

$$
\begin{aligned}
& +\sum_{j=1}^{n}\left|b_{i j}(s)\right| L_{j}\left|x_{j}(s)-y_{j}(s)\right| \\
& +\sum_{j=1}^{n}\left|c_{i j}(s)\right| L_{j} \\
& \left.\times\left|x_{j}\left(s-\tau_{i j}\right)-y_{j}\left(s-\tau_{i j}\right)\right|\right] d s .
\end{aligned}
$$

(16)

It immediately follows that

$$
\begin{aligned}
& e^{-K t}\left|x_{i}(t)-y_{i}(t)\right| \\
& \leq e^{-K t}\left|\phi_{i}(0)-\varphi_{i}(0)\right|+\frac{1}{\Gamma(\alpha)} e^{-K t} \\
& \times \int_{0}^{t}(t-s)^{\alpha-1} \\
& \times\left[\sum_{j=1}^{n}\left|a_{i j}(s)\right|\left|x_{j}(s)-y_{j}(s)\right|\right. \\
& +\sum_{j=1}^{n}\left|b_{i j}(s)\right| L_{j}\left|x_{j}(s)-y_{j}(s)\right| \\
& +\sum_{j=1}^{n}\left|c_{i j}(s)\right| L_{j} \\
& \left.\times\left|x_{j}\left(s-\tau_{i j}\right)-y_{j}\left(s-\tau_{i j}\right)\right|\right] d s \\
& \leq e^{-K t}\left|\phi_{i}(0)-\varphi_{i}(0)\right|+\frac{1}{\Gamma(\alpha)} \\
& \times \int_{0}^{t}(t-s)^{\alpha-1} \\
& \times\left[\sum_{j=1}^{n}\left|a_{i j}(s)\right| e^{-K(t-s)} e^{-K s}\left|x_{j}(s)-y_{j}(s)\right|\right. \\
& +\sum_{j=1}^{n}\left|b_{i j}(s)\right| L_{j} e^{-K(t-s)} \\
& \times e^{-K s}\left|x_{j}(s)-y_{j}(s)\right| \\
& +\sum_{j=1}^{n}\left|c_{i j}(s)\right| L_{j} e^{-K\left(t-\left(s-\tau_{i j}\right)\right)} e^{-K\left(s-\tau_{i j}\right)} \\
& \left.\times\left|x_{j}\left(s-\tau_{i j}\right)-y_{j}\left(s-\tau_{i j}\right)\right|\right] d s \\
& \leq e^{-K t}\left|\phi_{i}(0)-\varphi_{i}(0)\right|+\frac{\sup _{t, \forall j}\left|a_{i j}(t)\right|}{\Gamma(\alpha)}
\end{aligned}
$$

$$
\begin{aligned}
& \times \int_{0}^{t}(t-s)^{\alpha-1} \sum_{j=1}^{n} e^{-K(t-s)} e^{-K s}\left|x_{j}(s)-y_{j}(s)\right| d s \\
& +\frac{\sup _{t, \forall j}\left|b_{i j}(t)\right|}{\Gamma(\alpha)} L \int_{0}^{t}(t-s)^{\alpha-1} \\
& \times \sum_{j=1}^{n} e^{-K(t-s)} e^{-K s}\left|x_{j}(s)-y_{j}(s)\right| d s \\
& +\frac{\sup _{t, \forall j}\left|c_{i j}(t)\right|}{\Gamma(\alpha)} L \\
& \times \int_{0}^{t}(t-s)^{\alpha-1} \sum_{j=1}^{n} e^{-K\left(t-\left(s-\tau_{i j}\right)\right)} e^{-K\left(s-\tau_{i j}\right)} \\
& \times\left|x_{j}\left(s-\tau_{i j}\right)-y_{j}\left(s-\tau_{i j}\right)\right| d s \\
& \leq e^{-K t}\left|\phi_{i}(0)-\varphi_{i}(0)\right|+\frac{\sup _{t, \forall j}\left|a_{i j}(t)\right|}{\Gamma(\alpha)} \\
& \times \int_{0}^{t}(t-s)^{\alpha-1} \\
& \times \sum_{j=1}^{n} e^{-K(t-s)} e^{-K s}\left|x_{j}(s)-y_{j}(s)\right| d s \\
& +\frac{\sup _{t, \forall j}\left|b_{i j}(t)\right|}{\Gamma(\alpha)} L \\
& \times \int_{0}^{t}(t-s)^{\alpha-1} \\
& \times \sum_{j=1}^{n} e^{-K(t-s)} e^{-K s}\left|x_{j}(s)-y_{j}(s)\right| d s \\
& +\frac{\sup _{t, \forall j}\left|c_{i j}(t)\right|}{\Gamma(\alpha)} L \\
& \times \sum_{j=1}^{n} \int_{0}^{t-\tau_{i j}}\left(t-\tau_{i j}-\theta\right)^{\alpha-1} \\
& \times e^{-K(t-\theta)} e^{-K \theta}\left|x_{j}(\theta)-y_{j}(\theta)\right| d \theta \\
& +\frac{\sup _{t, \forall j}\left|c_{i j}(t)\right|}{\Gamma(\alpha)} L \sum_{j=1}^{n} \int_{-\tau_{i j}}^{0}\left(t-\tau_{i j}-\theta\right)^{\alpha-1} e^{-K(t-\theta)} \\
& \times e^{-K \theta}\left|\phi_{j}(\theta)-\varphi_{j}(\theta)\right| d \theta \\
& \leq \sup _{t \in[-\tau, 0]}\left\{e^{-K t}\left|\phi_{i}(t)-\varphi_{i}(t)\right|\right\} \\
& +a_{i} \sum_{j=1}^{n} \sup _{t}\left\{e^{-K t}\left|x_{j}(t)-y_{j}(t)\right|\right\} \frac{1}{\Gamma(\alpha)} \\
& \times \int_{0}^{t}(t-s)^{\alpha-1} e^{-K(t-s)} d s
\end{aligned}
$$




$$
\begin{aligned}
& +b_{i} L \sum_{j=1}^{n} \sup _{t}\left\{e^{-K t}\left|x_{j}(t)-y_{j}(t)\right|\right\} \frac{1}{\Gamma(\alpha)} \\
& \times \int_{0}^{t}(t-s)^{\alpha-1} e^{-K(t-s)} d s \\
& +c_{i} L \sum_{j=1}^{n} \sup _{t}\left\{e^{-K t}\left|x_{j}(t)-y_{j}(t)\right|\right\} \frac{1}{\Gamma(\alpha)} \\
& \times \int_{0}^{t-\tau_{i j}}\left(t-\tau_{i j}-\theta\right)^{\alpha-1} e^{-K(t-\theta)} d \theta \\
& +c_{i} L \sum_{j=1}^{n} \sup _{t \in[-\tau, 0]}\left\{e^{-K t}\left|\phi_{j}(t)-\varphi_{j}(t)\right|\right\} \frac{1}{\Gamma(\alpha)} \\
& \times \int_{-\tau_{i j}}^{0}\left(t-\tau_{i j}-\theta\right)^{\alpha-1} e^{-K(t-\theta)} d \theta \\
& <\sup _{t \in[-\tau, 0]}\left\{e^{-K t}\left|\phi_{i}(t)-\varphi_{i}(t)\right|\right\} \\
& +\frac{a_{i}}{K^{\alpha}} \sum_{j=1}^{n} \sup _{t}\left\{e^{-K t}\left|x_{j}(t)-y_{j}(t)\right|\right\} \\
& +\frac{b_{i} L}{K^{\alpha}} \sum_{j=1}^{n} \sup _{t}\left\{e^{-K t}\left|x_{j}(t)-y_{j}(t)\right|\right\} \\
& +\frac{c_{i} L}{K^{\alpha}} \sum_{j=1}^{n} \sup _{t}\left\{e^{-K t}\left|x_{j}(t)-y_{j}(t)\right|\right\} \\
& +\frac{c_{i} L}{K^{\alpha}} \sum_{j=1}^{n} \sup _{t \in[-\tau, 0]}\left\{e^{-K t}\left|\phi_{j}(t)-\varphi_{j}(t)\right|\right\} \\
& =\sup _{t \in[-\tau, 0]}\left\{e^{-K t}\left|\phi_{i}(t)-\varphi_{i}(t)\right|\right\} \\
& +\frac{c_{i} L}{K^{\alpha}}\|\phi(t)-\varphi(t)\|+\frac{a_{i}+b_{i} L+c_{i} L}{K^{\alpha}}\|x(t)-y(t)\|,
\end{aligned}
$$

which implies

$$
\begin{aligned}
\|x(t)-y(t)\| \\
=\sum_{i=1}^{n} \sup _{t}\left\{e^{-K t}\left|x_{i}(t)-y_{i}(t)\right|\right\} \\
<\sum_{i=1}^{n}\left(\sup _{t[-\tau, 0]}\left\{e^{-K t}\left|\phi_{i}(t)-\varphi_{i}(t)\right|\right\}\right. \\
+\frac{c_{i} L}{K^{\alpha}}\|\phi(t)-\varphi(t)\| \\
\left.+\frac{a_{i}+b_{i} L+c_{i} L}{K^{\alpha}}\|x(t)-y(t)\|\right)
\end{aligned}
$$

$$
\begin{aligned}
= & \left(1+\frac{\|C\| L}{K^{\alpha}}\right)\|\phi(t)-\varphi(t)\| \\
& +\frac{\|A\|+(\|B\|+\|C\|) L}{K^{\alpha}}\|x(t)-y(t)\| .
\end{aligned}
$$

Because $K$ is a large enough constant, now we choose $K$ large enough constant such that $\|A\|+(\|B\|+\|C\|) L<K^{\alpha}$; then, from (18), we know that

$$
\|x(t)-y(t)\|<\frac{K^{\alpha}+\|C\| L}{K^{\alpha}-(\|A\|+(\|B\|+\|C\|) L)}\|\phi(t)-\varphi(t)\| .
$$

Therefore, for any $\varepsilon>0$, there exists $\delta(\varepsilon)=\left(\left(K^{\alpha}-(\|A\|+\right.\right.$ $\left.(\|B\|+\|C\|) L)) /\left(K^{\alpha}+\|C\| L\right)\right) \varepsilon>0$ such that $\|x(t)-y(t)\|<$ $\varepsilon$ when $\|\phi(t)-\varphi(t)\|<\delta$. According to Definition 5, the solution of system (6) is uniformly stable.

Remark 7. Reference [16] investigated the uniform stability and the existence and uniqueness of solutions of a class of fractional-order nonautonomous systems (or differential equations), respectively, but without considering the time delay.

Remark 8. To the best of our knowledge, the theoretical result on stability analysis of fractional-order nonlinear systems with multiple time delays has not been seen yet.

\section{Illustrative Examples}

In this section, we consider two examples to illustrate the obtained results.

Example 1. Consider the following fractional system with time-invariant coefficients and multiple time delays:

$$
\begin{aligned}
D^{\alpha} x_{1}(t)= & -1.3 x_{1}(t)+0.9 x_{2}(t)-0.5 \tanh \left(x_{1}(t)\right) \\
& +\tanh \left(x_{2}(t)\right)-0.5 \tanh \left(x_{1}(t-0.01)\right) \\
& +0.6 \tanh \left(x_{2}(t-0.02)\right)-1.8 \\
D^{\alpha} x_{2}(t)= & 1.2 x_{1}(t)-1.6 x_{2}(t)-0.4 \tanh \left(x_{1}(t)\right) \\
& +0.1 \tanh \left(x_{2}(t)\right)-\tanh \left(x_{1}(t-0.02)\right) \\
& -1.9 \tanh \left(x_{2}(t-0.01)\right)+2
\end{aligned}
$$

where $\alpha=0.9, T<+\infty$, with an associated function of the initial state:

$$
\phi_{1}(t)=2 \cos t, \quad \phi_{2}(t)=\sin t-1, \quad t \in[-0.02,0] .
$$

Since function $\tanh (x)$ is Lipschitz continuous $\left(L_{1}=\right.$ $L_{2}=1$ ), then the condition of the theorems is satisfied. Hence, by Theorems 4 and 6, we conclude that system (20) has a unique solution, which is uniformly stable. 


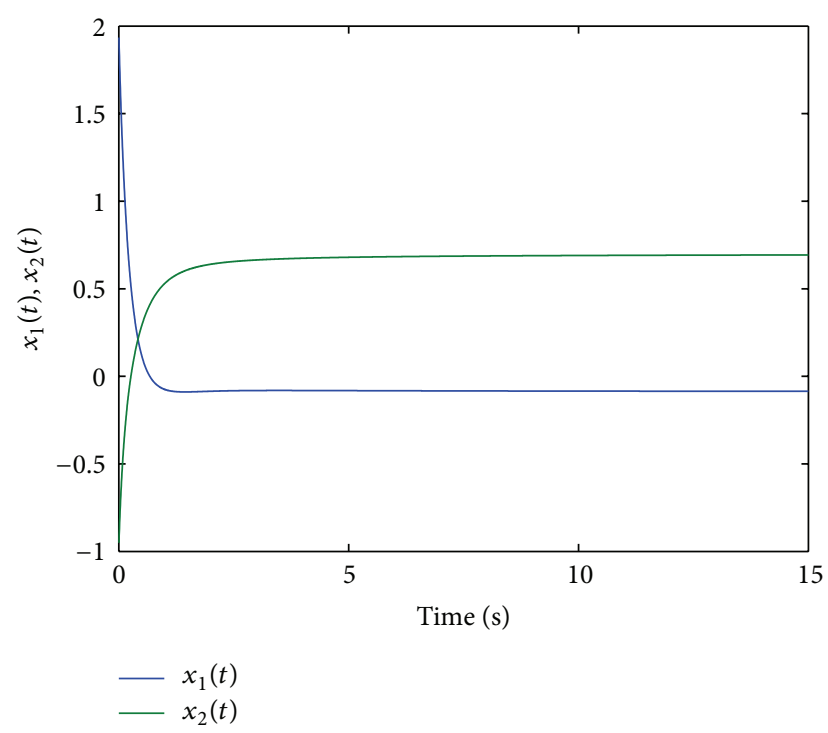

FIgURE 1: The dynamic behavior of system (20).

In fact, system (20) has a unique fixed point $x^{*}=$ $\left(x_{1}^{*}, x_{2}^{*}\right)^{T}$, which satisfies

$$
\begin{aligned}
& -1.3 x_{1}^{*}+0.9 x_{2}^{*}-\tanh \left(x_{1}^{*}\right)+1.6 \tanh \left(x_{2}^{*}\right)-1.8=0, \\
& 1.2 x_{1}^{*}-1.6 x_{2}^{*}-1.5 \tanh \left(x_{1}^{*}\right)-1.5 \tanh \left(x_{2}^{*}\right)+2=0 .
\end{aligned}
$$

By calculation, the unique fixed point $x^{*}=\left(x_{1}^{*}, x_{2}^{*}\right)^{T}$ of system $(20)$ is $(-0.088,0.670)$. Figure 1 shows that the solution of system (20) converges to the fixed point $x^{*}$.

Example 2. Consider the following fractional delay system with time-varying coefficients:

$$
\begin{aligned}
D^{\alpha} x_{1}(t)= & \frac{1}{5} e^{-t} x_{1}(t)-\frac{1}{6} \sin t \times x_{2}(t) \\
& +\frac{3}{10} \tanh \left(x_{1}(t)\right)-\frac{1+\cos t}{8} \tanh \left(x_{2}(t)\right) \\
& -\frac{1}{8} \tanh \left(x_{1}(t-0.03)\right) \\
& +\frac{1}{9} \tanh \left(x_{2}(t-0.01)\right)+\sin ^{2} t, \\
D^{\alpha} x_{2}(t)= & \frac{1}{10} \cos t \times x_{1}(t)+\frac{1}{10} e^{-2 t} x_{2}(t) \\
& -\frac{1}{10} \tanh \left(x_{1}(t)\right)+\frac{1}{12} e^{-t} \tanh \left(x_{2}(t)\right) \\
& +\frac{1}{6} \sin t \times \tanh \left(x_{1}(t-0.01)\right) \\
& +\frac{1}{8} \tanh \left(x_{2}(t-0.02)\right)-\cosh (t),
\end{aligned}
$$

where $0<\alpha<1, T<+\infty$, with an associated function of the initial state:

$$
\begin{array}{r}
\phi_{1}(t)=\left|x+\frac{3}{2}\right|-\left|x-\frac{1}{2}\right|, \quad \phi_{2}(t)=1-x^{2}, \\
t \in[-0.03,0] .
\end{array}
$$

From (23), one can obtain

$$
\begin{gathered}
A(t)=\left(\begin{array}{cc}
\frac{1}{5} e^{-t} & -\frac{1}{6} \sin t \\
\frac{1}{10} \cos t & \frac{1}{10} e^{-2 t}
\end{array}\right), \\
B(t)=\left(\begin{array}{cc}
\frac{3}{10} & -\frac{1+\cos t}{8} \\
-\frac{1}{10} & \frac{1}{12} e^{-t}
\end{array}\right), \\
C(t)=\left(\begin{array}{cc}
-\frac{1}{8} & \frac{1}{9} \\
\frac{1}{6} \sin t & \frac{1}{8}
\end{array}\right) \\
u(t)=\left(\begin{array}{c}
\sin ^{2} t \\
-\cosh (t)
\end{array}\right) .
\end{gathered}
$$

Since function $\tanh (x)$ is a Lipschitz continuous function, then according to Theorems 4 and 6 , we conclude that system (23) has a unique uniformly stable solution.

\section{Conclusion}

Uniform stability problem of a class of fractional-order nonautonomous systems with multiple time delays is discussed in this paper. Moreover, the existence and uniqueness of solutions under certain conditions are proven by using Banach fixed point principle. At last, two examples are given for illustration.

\section{Conflict of Interests}

The authors declare that there is no conflict of interests regarding the publication of this paper.

\section{References}

[1] D. D. Demir, N. Bildik, and B. G. Sinir, "Application of fractional calculus in the dynamics of beams," Boundary Value Problems, vol. 2012, article 135, 2012.

[2] M. A. García-González, M. Fernández-Chimeno, L. Capdevila, E. Parrado, and J. Ramos-Castro, "An application of fractional differintegration to heart rate variability time series," Computer Methods and Programs in Biomedicine, vol. 111, no. 1, pp. 33-40, 2013.

[3] Z. Wang and X. Ma, "Application of fractional-order calculus approach to signal processing," in Proceedings of the 6th IEEE Joint International Information Technology and Artificial Intelligence Conference (ITAIC '11), vol. 1, pp. 220-222, IEEE, 2011. 
[4] I. S. Jesus and J. A. T. Machado, "Application of fractional order concepts in the study of electrical potential," in Dynamics, Games and Science. II, pp. 467-470, Springer, Heidelberg, Germany, 2011.

[5] D. A. Benson, M. M. Meerschaert, and J. Revielle, "Fractional calculus in hydrologic modeling: a numerical perspective," Advances in Water Resources, vol. 51, pp. 479-497, 2012.

[6] I. B. Bapna and N. Mathur, "Application of fractional calculus in statistics," International Journal of Contemporary Mathematical Sciences, vol. 7, no. 17-20, pp. 849-856, 2012.

[7] H. Zhang, J. Cao, and W. Jiang, "General solution of linear fractional neutral differential difference equations," Discrete Dynamics in Nature and Society, vol. 2013, Article ID 489521, 7 pages, 2013.

[8] C. Song, T. Zhu, and J. Cao, "Existence of solutions for fractional-order neutral differential inclusions with impulsive and nonlocal conditions," Discrete Dynamics in Nature and Society, vol. 2012, Article ID 363562, 14 pages, 2012.

[9] J. A. Tenreiro Machado, "Time-delay and fractional derivatives," Advances in Difference Equations, vol. 2011, Article ID 934094, 2011.

[10] Z. Jiao and Y. Zhong, "Robust stability for fractional-order systems with structured and unstructured uncertainties," Computers \& Mathematics with Applications, vol. 64, no. 10, pp. 3258-3266, 2012.

[11] J. Lu, Y. Chen, and W. Chen, "Robust asymptotical stability of fractionalorder linear systems with structured perturbations," Computers \& Mathematics with Applications, vol. 66, no. 5, pp. 873-882, 2013.

[12] H.-S. Ahn and Y. Chen, "Necessary and sufficient stability condition of fractional-order interval linear systems," Automatica, vol. 44, no. 11, pp. 2985-2988, 2008.

[13] Y. Li, Y. Chen, and I. Podlubny, "Mittag-Leffler stability of fractional order nonlinear dynamic systems," Automatica, vol. 45, no. 8, pp. 1965-1969, 2009.

[14] Z. Jiao and Y. Q. Chen, "Stability of fractional-order linear timeinvariant systems with multiple noncommensurate orders," Computers \& Mathematics with Applications, vol. 64, no. 10, pp. 3053-3058, 2012.

[15] N. Tan, Ö. Faruk Özgüven, and M. Mine Özyetkin, "Robust stability analysis of fractional order interval polynomials," ISA Transactions, vol. 48, no. 2, pp. 166-172, 2009.

[16] A. M. A. El-Sayed and Sh. A. Abd El-Salam, "On the stability of a fractional-order differential equation with nonlocal initial condition," Electronic Journal of Qualitative Theory of Differential Equations, vol. 29, pp. 1-8, 2008.

[17] M. P. Lazarević and A. M. Spasić, "Finite-time stability analysis of fractional order time-delay systems: Gronwall's approach," Mathematical and Computer Modelling, vol. 49, no. 3-4, pp. 475481, 2009.

[18] C. Zeng, Y. Chen, and Q. Yang, "Robust controllability of interval fractional order linear time invariant stochastic systems," in Proceedings of the IEEE 51st Annual Conference on Decision and Control (CDC '12), pp. 4047-4050, IEEE, 2012.

[19] H. Shao, "New delay-dependent stability criteria for systems with interval delay," Automatica, vol. 45, no. 3, pp. 744-749, 2009.

[20] X. Meng, J. Lam, B. Du, and H. Gao, "A delay-partitioning approach to the stability analysis of discrete-time systems," Automatica, vol. 46, no. 3, pp. 610-614, 2010.
[21] C. W. Chen, "Stability analysis and robustness design of nonlinear systems: an nn-based approach," Applied Soft Computing, vol. 11, pp. 2735-2742, 2011.

[22] F. Qiu, B. Cui, and Y. Ji, "Further results on robust stability of neutral system with mixed time-varying delays and nonlinear perturbations," Nonlinear Analysis: Real World Applications, vol. 11, no. 2, pp. 895-906, 2010.

[23] K. Akbari Moornani and M. Haeri, "On robust stability of LTI fractional-order delay systems of retarded and neutral type," Automatica, vol. 46, no. 2, pp. 362-368, 2010.

[24] S. Kumar and N. Sukavanam, "Approximate controllability of fractional order semilinear systems with bounded delay," Journal of Differential Equations, vol. 252, no. 11, pp. 6163-6174, 2012.

[25] Z. Wang, X. Huang, and G. Shi, "Analysis of nonlinear dynamics and chaos in a fractional order financial system with time delay," Computers \& Mathematics with Applications, vol. 62, no. 3, pp. 1531-1539, 2011.

[26] A. M. El-Sayed and F. M. Gaafar, "Stability of a nonlinear nonautonomous fractional order systems with different delays and non-local conditions," Advances in Difference Equations, vol. 2011, article 47, 2011.

[27] A. M. El-Sayed, F. M. Gaafar, and E. M. Hamadalla, "Stability for a nonlocal nonlocal non-autonomous system of fractional order differential equations with delays," Electronic Journal of Differential Equations, vol. 2010, pp. 1-10, 2010.

[28] C. Song, "Stability analysis of fractional order hopfield neural networks with delays," American Journal of Engineering and Technology Research, vol. 11, no. 12.

[29] H. Zhang, J. Cao, and W. Jiang, "Controllability criteria for linear fractional differential systems with state delay and impulses," Journal of Applied Mathematics, vol. 2013, Article ID 146010, 9 pages, 2013.

[30] J. Shen and J. Cao, "Necessary and sufficient conditions for consensus of delayed fractional-order systems," Asian Journal of Control, vol. 14, no. 6, pp. 1690-1697, 2012.

[31] S. G. Samko, A. A. Kilbas, and O. I. Marichev, Fractional Integrals and Derivatives, Gordon and Breach Science Publishers, New York, NY, USA, 1993.

[32] I. Podlubny, Fractional Differential Equations, vol. 198 of Mathematics in Science and Engineering, Academic Press, San Diego, Calif, USA, 1999.

[33] C. Corduneanu, Principles of Differential and Integral Equations, Chelsea Publishing, New York, NY, USA, 2nd edition, 1977. 


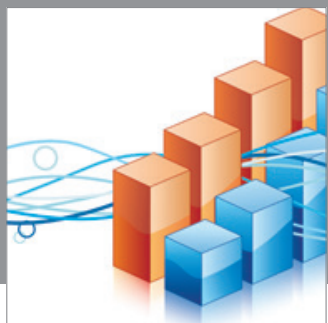

Advances in

Operations Research

mansans

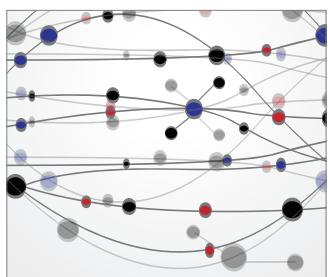

The Scientific World Journal
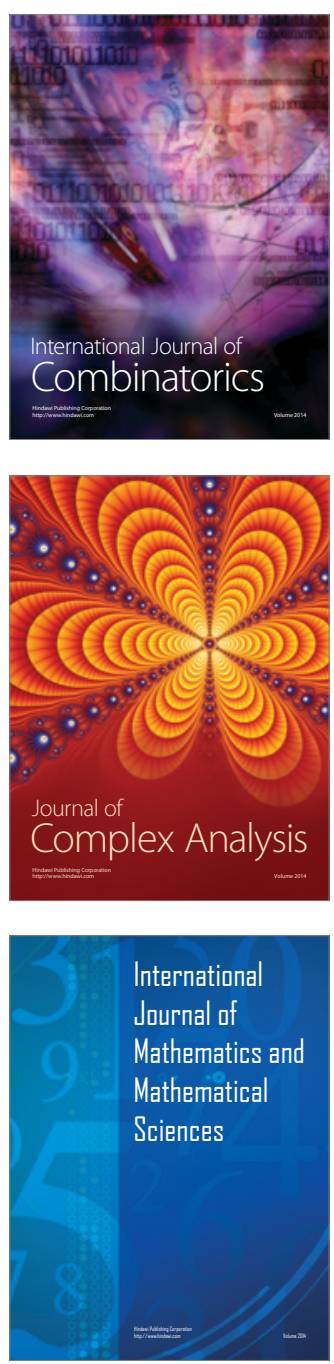
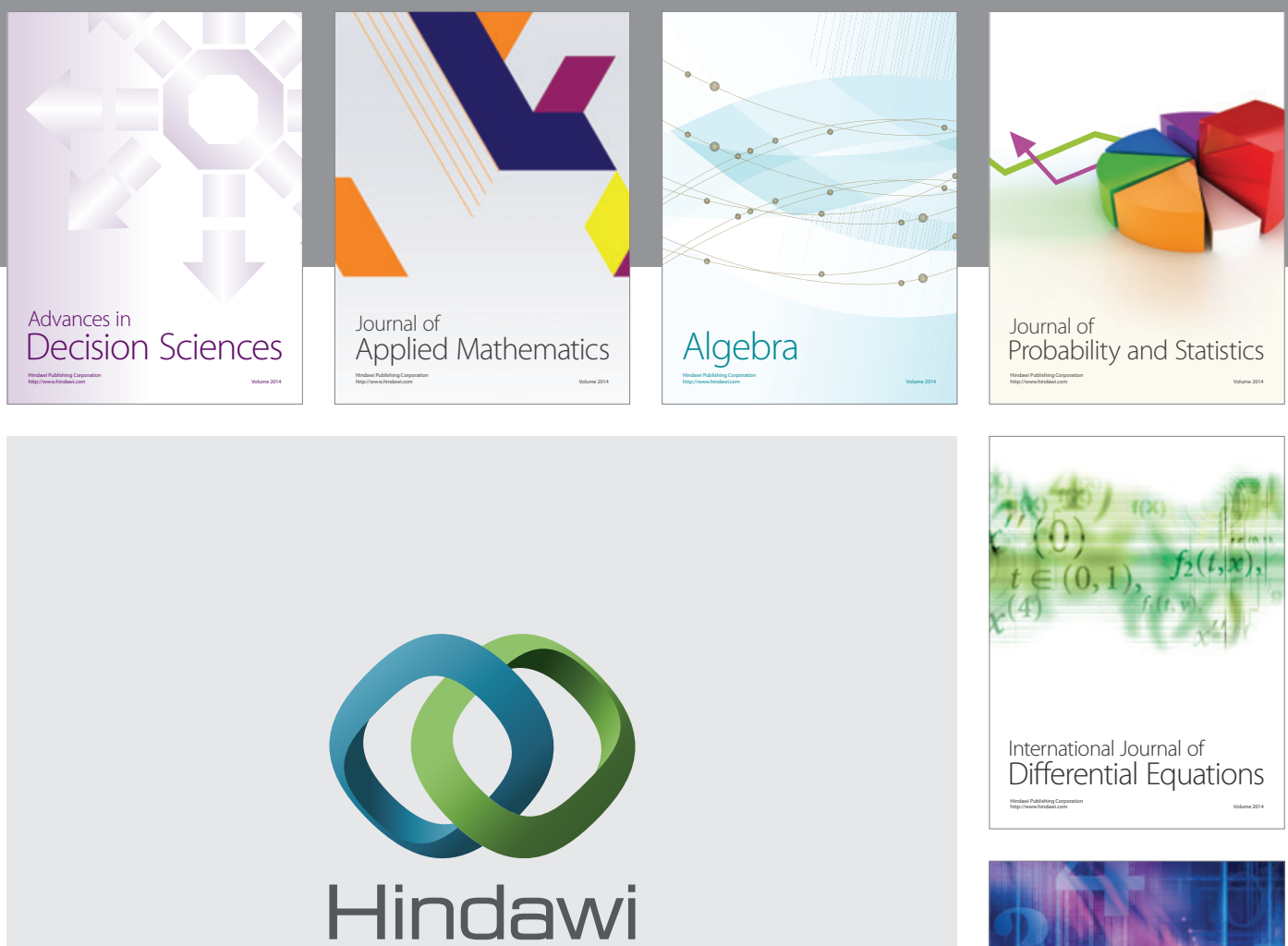

Submit your manuscripts at http://www.hindawi.com
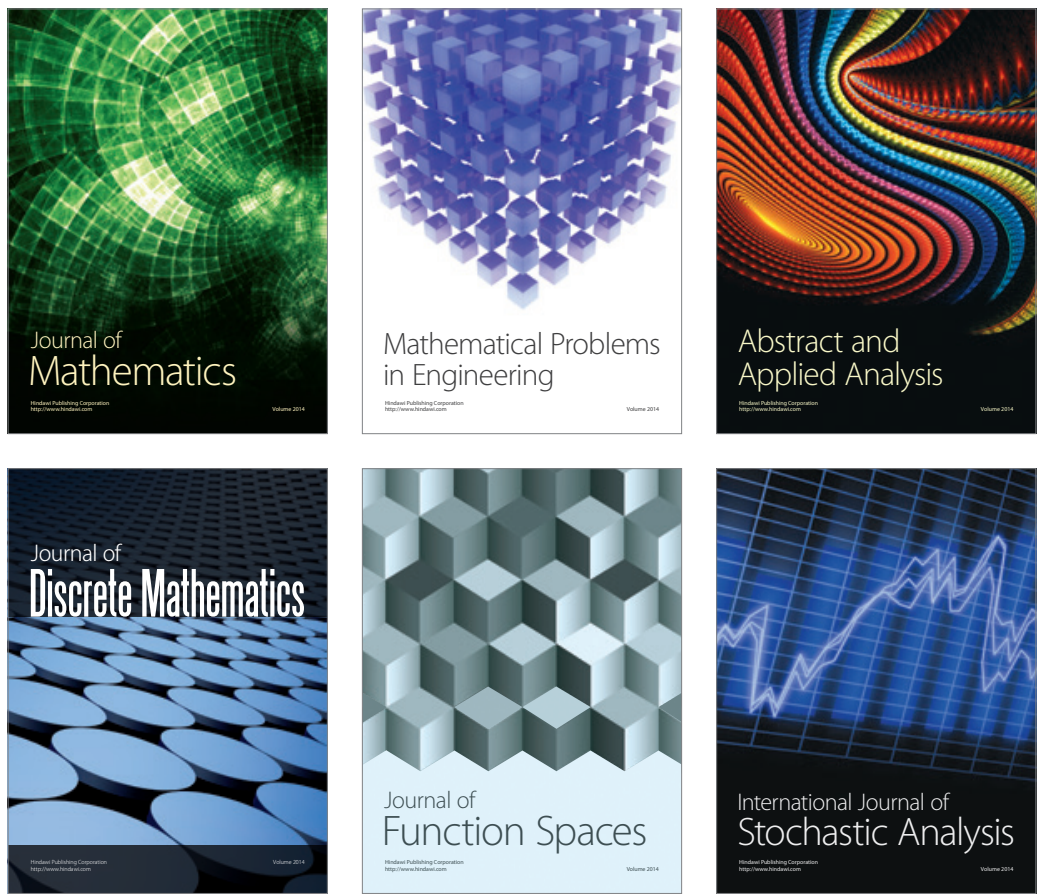

Journal of

Function Spaces

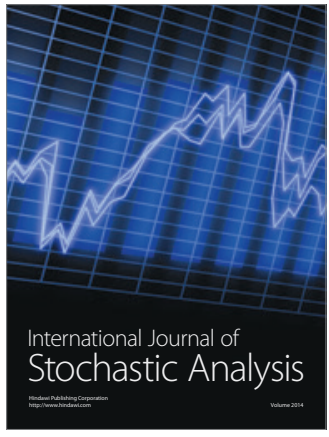

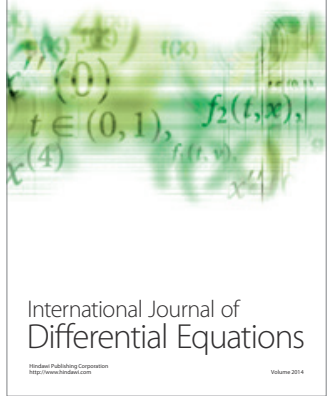
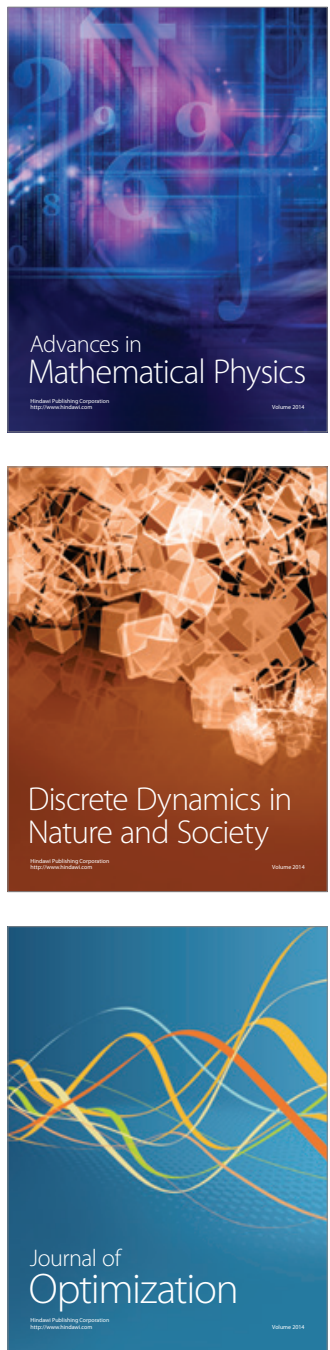